PROCEEDINGS OF THE

AMERICAN MATHEMATICAL SOCIETY

Volume 131, Number 1, Pages 87-94

S 0002-9939(02)06635-2

Article electronically published on May 22, 2002

\title{
THE $a$-INVARIANT AND GORENSTEINNESS OF GRADED RINGS ASSOCIATED TO FILTRATIONS OF IDEALS IN REGULAR LOCAL RINGS
}

\author{
SHIRO GOTO, FUTOSHI HAYASAKA, AND SHIN-ICHIRO IAI \\ (Communicated by Wolmer V. Vasconcelos)
}

\begin{abstract}
Let $A$ be a regular local ring and let $\mathcal{F}=\left\{F_{n}\right\}_{n \in \mathbb{Z}}$ be a filtration of ideals in $A$ such that $\mathcal{R}(\mathcal{F})=\bigoplus_{n \geq 0} F_{n}$ is a Noetherian ring with $\operatorname{dim} \mathcal{R}(\mathcal{F})=$ $\operatorname{dim} A+1$. Let $\mathcal{G}(\mathcal{F})=\bigoplus_{n \geq 0} F_{n} / F_{n+1}$ and let a $(\mathcal{G}(\mathcal{F}))$ be the $a$-invariant of $\mathcal{G}(\mathcal{F})$. Then the theorem says that $F_{1}$ is a principal ideal and $F_{n}=F_{1}^{n}$ for all $n \in \mathbb{Z}$ if and only if $\mathcal{G}(\mathcal{F})$ is a Gorenstein ring and a $(\mathcal{G}(\mathcal{F}))=-1$. Hence $\mathrm{a}(\mathcal{G}(\mathcal{F})) \leq-2$, if $\mathcal{G}(\mathcal{F})$ is a Gorenstein ring, but the ideal $F_{1}$ is not principal.
\end{abstract}

\section{INTRODUCTION}

Let $A$ be a Noetherian local ring with the maximal ideal $\mathfrak{m}$ and $d=\operatorname{dim} A$. Let $\mathcal{F}=\left\{F_{n}\right\}_{n \in \mathbb{Z}}$ be a family of ideals in $A$. Then we say that $\mathcal{F}$ is a filtration of ideals in $A$, if it satisfies the following conditions:

(1) $F_{n} \supseteq F_{n+1}$ for all $n \in \mathbb{Z}$,

(2) $F_{0}=A \supsetneq F_{1}$, and

(3) $F_{m} F_{n} \subseteq F_{m+n}$ for all $m, n \in \mathbb{Z}$.

For each filtration $\mathcal{F}$ of ideals in $A$ we define

$$
\begin{aligned}
& \mathcal{R}(\mathcal{F})=\sum_{n \geq 0} F_{n} t^{n} \subseteq A[t], \\
& \mathcal{R}^{\prime}(\mathcal{F})=\sum_{n \in \mathbb{Z}} F_{n} t^{n} \subseteq A\left[t, t^{-1}\right], \text { and } \\
& \mathcal{G}(\mathcal{F})=\mathcal{R}^{\prime}(\mathcal{F}) / t^{-1} \mathcal{R}^{\prime}(\mathcal{F})
\end{aligned}
$$

(here $t$ denotes an indeterminate over $A$ ), which we call the Rees algebra, the extended Rees algebra, and the associated graded ring of $\mathcal{F}$. We say that $\mathcal{F}$ is a $\operatorname{good}$ filtration if $\mathcal{R}(\mathcal{F})$ is a Noetherian $\operatorname{ring}$ and $\operatorname{dim} \mathcal{R}(\mathcal{F})=\operatorname{dim} A+1$. If $\mathcal{R}(\mathcal{F})$ is a Noetherian ring, the latter condition is equivalent to saying that $F_{1} \not \subset \bigcap_{\mathfrak{p} \in \operatorname{Assh} A} \mathfrak{p}$, where $\operatorname{Assh} A=\{\mathfrak{p} \in \operatorname{Spec} A \mid \operatorname{dim} A / \mathfrak{p}=\operatorname{dim} A\}$ ([GNi, Part II, Lemma (2.2)]). When the filtration $\mathcal{F}$ is ideal-adic, that is, $F_{n}=I^{n}$ for all $n \in \mathbb{Z}$ for some ideal $I$ in $A$, we denote $\mathcal{R}(\mathcal{F}), \mathcal{R}^{\prime}(\mathcal{F})$, and $\mathcal{G}(\mathcal{F})$ by $\mathcal{R}(I), \mathcal{R}^{\prime}(I)$, and $\mathcal{G}(I)$, respectively.

Let $\mathcal{F}$ be a good filtration of ideals in $A$. Let $\mathfrak{M}=\mathfrak{m} \mathcal{G}(\mathcal{F})+[\mathcal{G}(\mathcal{F})]_{+}$and let $\mathrm{H}_{\mathfrak{M}}^{d}(\mathcal{G}(\mathcal{F}))$ denote the $d^{\underline{t h}}$ local cohomology module of $\mathcal{G}(\mathcal{F})$ with respect to the

Received by the editors August 25, 2001.

2000 Mathematics Subject Classification. Primary 13H05; Secondary 13H10.

Key words and phrases. Injective dimension, integrally closed ideal, $\mathfrak{m}$-full ideal, regular local ring, Gorenstein local ring, $a$-invariant, Rees algebra, associated graded ring, filtration of ideals.

The first author was supported by the Grant-in-Aid for Scientific Researches in Japan $(\mathrm{C}(2)$, No. 11640049). 
graded maximal ideal $\mathfrak{M}$ in $\mathcal{G}(\mathcal{F})$. We put

$$
\mathrm{a}(\mathcal{G}(\mathcal{F}))=\max \left\{n \in \mathbb{Z} \mid\left[\mathrm{H}_{\mathfrak{M}}^{d}(\mathcal{G}(\mathcal{F}))\right]_{n} \neq(0)\right\}
$$

(GW, Definition (3.1.4)]) and call it the $a$-invariant of $\mathcal{G}(\mathcal{F})$. Let $\mathrm{K}_{\mathcal{R}(\mathcal{F})}$ and $\mathrm{K}_{\mathcal{R}^{\prime}(\mathcal{F})}$ denote the canonical modules of $\mathcal{R}(\mathcal{F})$ and $\mathcal{R}^{\prime}(\mathcal{F})$ respectively (cf. [HK], GNi, HIO ). With this notation the main result of this paper is stated as follows.

Theorem (1.1). Let $A$ be a Gorenstein local ring and let $\mathcal{F}=\left\{F_{n}\right\}_{n \in \mathbb{Z}}$ be a good filtration of ideals in $A$. Then the following three conditions are equivalent:

(1) $\mathcal{R}^{\prime}(\mathcal{F})$ is a Gorenstein ring and $\mathrm{K}_{\mathcal{R}^{\prime}(\mathcal{F})} \cong \mathcal{R}^{\prime}(\mathcal{F})$ as graded $\mathcal{R}^{\prime}(\mathcal{F})$-modules.

(2) $\mathcal{R}(\mathcal{F})$ is a Cohen-Macaulay ring and $\mathrm{K}_{\mathcal{R}(\mathcal{F})} \cong[\mathcal{R}(\mathcal{F})]_{+}$as graded $\mathcal{R}(\mathcal{F})$ modules.

(3) $\mathcal{G}(\mathcal{F})$ is a Gorenstein ring and $\mathrm{a}(\mathcal{G}(\mathcal{F}))=-1$.

If $A$ is furthermore a regular local ring, one may add the following:

(4) $\mathcal{R}(\mathcal{F})$ is a regular ring.

(5) $\mathcal{R}(\mathcal{F})$ is the polynomial ring in one variable over $A$.

(6) $F_{1}$ is a principal ideal and $F_{n}=F_{1}^{n}$ for all $n \in \mathbb{Z}$.

The equivalence of conditions (1), (2), and (3) in Theorem (1.1) is partially reported by [GIW, Theorem (6.1)] and GK Proposition (4.1)].

Since $\mathrm{a}(\mathcal{G}(\mathcal{F}))<0([\underline{\mathrm{L}})$, as a consequence of Theorem (1.1) we have the following.

Corollary (1.2). Let $A$ be a regular local ring and let $\mathcal{F}=\left\{F_{n}\right\}_{n \in \mathbb{Z}}$ be a good filtration of ideals in $A$. Then $\mathrm{a}(\mathcal{G}(\mathcal{F})) \leq-2$, if $\mathcal{G}(\mathcal{F})$ is a Gorenstein ring and $F_{1}$ is not a principal ideal.

Unless $A$ is regular, the inequality a $(\mathcal{G}(\mathcal{F})) \leq-2$ in Corollary (1.2) is no longer true, even though $A$ is a rational singularity (Remark (3.1)). We consult GIW Section 8] about the examples of $\mathfrak{m}$-primary ideals $I$ in the formal power series ring $A=k[[X, Y, Z]]$ in three variables over a field $k$ for which the $\operatorname{rings} \mathcal{G}(I)$ are Gorenstein and a $(\mathcal{G}(I))=-2$. See GNS1, GNS2 for the examples of filtrations $\mathcal{F}$ of ideals in a 3 -dimensional regular local ring $A$ such that the $\operatorname{rings} \mathcal{G}(\mathcal{F})$ are Gorenstein and $\mathrm{a}(\mathcal{G}(\mathcal{F}))=-2$, where the symbolic Rees algebras $\mathcal{R}_{s}(\mathfrak{p})$ of certain height 2 prime ideals $\mathfrak{p}$ in $A=k[[X, Y, Z]]$ are explored.

The $a$-invariant $\mathrm{a}(\mathcal{G}(\mathcal{F})$ ) has played a specific role in the analysis of the Cohen-Macaulayness and Gorensteinness of $\mathcal{R}(\mathcal{F})$. For instance, $\mathcal{R}(\mathcal{F})$ is a Cohen-Macaulay (resp. Gorenstein) ring if and only if $\mathcal{G}(\mathcal{F})$ is a Cohen-Macaulay ring with $\mathrm{a}(\mathcal{G}(\mathcal{F}))<0$ (resp. $\mathcal{G}(\mathcal{F})$ is a Gorenstein ring with a $(\mathcal{G}(\mathcal{F}))=-2$ ), provided that $A$ is a Cohen-Macaulay local ring (resp. $A$ is a Gorenstein local ring and $\left.\mathrm{ht}_{A} F_{1} \geq 2\right)$ (GNi, Part II]). This gives a generalization of the classical results due to [GS], I], and [TI]. However, one of the most striking achievements has been done by J. Lipman $[\mathbb{L}$, who proved that if $A$ is a pseudo-rational local ring, one always has a $(\mathcal{G}(\mathcal{F}))<0$ once $\mathcal{G}(\mathcal{F})$ is Cohen-Macaulay. Consequently, when $A$ is a regular local ring, $\mathcal{R}(\mathcal{F})$ is a Cohen-Macaulay ring if and only if $\mathcal{G}(\mathcal{F})$ is Cohen-Macaulay. Although Lipman [L] dealt with the ideal-adic case only, his proof still works for general good filtrations.

The present research originated with the question of what happens to the $a$ invariant $\mathrm{a}(\mathcal{G}(\mathcal{F}))$ in the case where $A$ is a regular local ring and $\mathcal{G}(\mathcal{F})$ is a Gorenstein ring. There is already given by Herrmann, Huneke, and Ribbe [HHR Theorem 2.5] a strong piece of evidence to guess that a $(\mathcal{G}(\mathcal{F})) \leq-2$ whenever $\mathcal{G}(\mathcal{F})$ is a Gorenstein ring and $F_{1}$ is not principal. They explored the case where $F_{n}=I^{n}$ for 
all $n \in \mathbb{Z}$ for some $\mathfrak{m}$-primary ideal $I$ in a regular local $\operatorname{ring} A$ with the infinite residue class field, and gave the inequality $\mathrm{r}_{\mathfrak{q}}(I)=\min \left\{0 \leq n \in \mathbb{Z} \mid I^{n+1}=\mathfrak{q} I^{n}\right\} \leq d-2$ for every minimal reduction $\mathfrak{q}$ of $I$, in the case where $d=\operatorname{dim} A \geq 2$ and $\mathcal{G}(I)$ is a Gorenstein ring. Hence $-d \leq \mathrm{a}(\mathcal{G}(I)) \leq-2$, because $\mathrm{a}(\mathcal{G}(I))=\mathrm{r}_{\mathfrak{q}}(I)-d$. Now, let $I$ be an arbitrary ideal of $h_{A} I=s \geq 2$ in a regular local ring $A$, and assume that $\mathcal{G}(I)$ is a Gorenstein ring. Then, letting $\mathfrak{p} \in \mathrm{V}(I)$ of $\mathrm{ht}_{A} \mathfrak{p}=s$, we get $\mathrm{a}(\mathcal{G}(I))=\mathrm{a}\left(A_{\mathfrak{p}} \otimes_{A} \mathcal{G}(I)\right)=\mathrm{a}\left(\mathcal{G}\left(I A_{\mathfrak{p}}\right)\right)$, since $\mathcal{G}(I)$ is a Gorenstein ring. Thus the bounds $-s \leq \mathrm{a}(\mathcal{G}(I)) \leq-2$ of a $(\mathcal{G}(I))$ follow from [HHR, Theorem 2.5], because $s=\operatorname{dim} A_{\mathfrak{p}} \geq 2$ and $I A_{\mathfrak{p}}$ is $\mathfrak{p} A_{\mathfrak{p}}$-primary. The assertion of the $\mathfrak{m}$-primary case is, therefore, sufficiently general as for the bounds of the $a$-invariants of Gorenstein graded rings associated to ideals. However, this argument does not work for the graded rings $\mathcal{G}(\mathcal{F})$ associated to general filtrations $\mathcal{F}$ of ideals, in order to see whether a $(\mathcal{G}(\mathcal{F})) \leq-2$ if $\mathcal{G}(\mathcal{F})$ is a Gorenstein ring and the ideal $F_{1}$ is not principal. Added to it, our proof of Theorem (1.1) is fairly simple and entirely different from that of $[\mathrm{HHR}]$. For this reason Theorem (1.1) and Corollary (1.2) might have some significance in the analysis of $a$-invariants.

Before entering the details, let us briefly explain how this paper is organized. The proof of Theorem (1.1) will be given in Section 3. Our proof is based on the analysis of prime ideals associated to integrally closed ideals of finite injective dimension (Theorem (2.1)). This enables us to show that any Cohen-Macaulay local ring $B$ must be regular if $B$ contains a canonical ideal $I$ for which the local ring $B / I$ is regular. We will apply this observation to the specific Cohen-Macaulay local ring $B=\mathcal{R}(\mathcal{F})_{\mathfrak{M}}$ with $\mathfrak{M}=\mathfrak{m} \mathcal{R}(\mathcal{F})+[\mathcal{R}(\mathcal{F})]_{+}$. Thereafter, our Theorem (1.1) will fairly directly follow from GI, Proposition 2.5], which explicitly describes the canonical module $\mathrm{K}_{\mathcal{R}(\mathcal{F})}$ of $\mathcal{R}(\mathcal{F})$ in terms of the canonical $\mathcal{F}$-filtration $\omega$ of $A$. In Section 4 we shall explore the Gorensteinness of $\mathcal{G}(I)$ for the ideals $I$ of small height in regular local rings $A$. We will confirm that $\mathcal{R}(I)$ is a Gorenstein ring if and only if $\mathcal{G}(I)$ is Gorenstein. Examples shall be given in order to show that every regular local ring $A$ of $\operatorname{dim} A \geq 3$ contains numerous non-Cohen-Macaulay ideals $I$ of ht ${ }_{A} I=2$ for which the rings $\mathcal{R}(I)$ and $\mathcal{G}(I)$ are Gorenstein.

\section{Finite injeCtive Dimension AND PRimes ASsociated TO INTEGRALLY CLOSED IDEALS}

The goal of this section is Corollary (2.4) below, which states that any CohenMacaulay local ring $A$ must be regular if $A$ contains a certain special canonical ideal $I$.

Let us begin with the following.

Theorem (2.1). Let $A$ be a Noetherian ring and let $I$ be an integrally closed ideal in $A$. Then the local ring $A_{\mathfrak{p}}$ is regular for all $\mathfrak{p} \in \operatorname{Ass}_{A} A / I$ if $\operatorname{id}_{A} I<\infty$.

Here $\operatorname{id}_{A} I$ denotes the injective dimension of $I$.

Proof. Passing to the local ring $A_{\mathfrak{p}}$, we may assume that the ring $(A, \mathfrak{m})$ is local and $\mathfrak{m} \in \operatorname{Ass}_{A} A / I$. We have to show that $A$ is a regular local ring. To do this, let $B=A[X]_{\mathfrak{m} A[X]}(X$ an indeterminate over $A)$ and $\varphi: A \rightarrow B$ be the canonical flat local homomorphism. Then, since all the fibers of $\varphi$ are regular, by [FT, Corollary 1] we have the equality $\operatorname{id}_{B} I B=\operatorname{id}_{A} I$. Therefore, because our target is the regularity of $A$, passing to the local ring $B$ we may assume without loss of generality that the residue class field $A / \mathfrak{m}$ of $A$ is infinite. Hence $I=\sqrt{(0)}$ or $I$ is $\mathfrak{m}$-full, that 
is, $\mathfrak{m} I: x=I$ for some $x \in \mathfrak{m}$ (cf. [G, Theorem (2.4)]). Let $I=\sqrt{(0)}$. Then $\operatorname{dim} A=0$ and $I=\mathfrak{m}$, since $\mathfrak{m} \in \operatorname{Ass}_{A} A / I$. Consequently, thanks to the classical result of Bass $[\bar{B}$, the $A$-module $\mathfrak{m}$ is injective, so that we have $\mathfrak{m}=(0)$, as it is a direct summand of $A$. Let $I \neq \sqrt{(0)}$. Then $I \neq(0)$ since $I$ is integrally closed, whence $\operatorname{id}_{A} I=\operatorname{depth} A$. Therefore $\operatorname{depth} A>0$ (recall that $(0) \neq I \subsetneq A$ ), so that by the proof of [G. Theorem (2.4)] we may choose the element $x \in \mathfrak{m}$ satisfying the equality $\mathfrak{m} I: x=I$ to be a non-zerodivisor in $A$. Then, since $A / \mathfrak{m}$ is a direct summand of $I / x I$ ([GH, Corollary (2.3)]) and since $\operatorname{id}_{A} I / x I<\infty$, we finally have that $\operatorname{id}_{A} A / \mathfrak{m}<\infty$, and so $A$ is a regular local ring.

Remark (2.2). The above proof shows that every Noetherian local ring A with the maximal ideal $\mathfrak{m}$ must be regular if $A$ contains an $\mathfrak{m}$-full ideal $I$ such that $\mathfrak{m} \in$ $\operatorname{Ass}_{A} A / I$ and $\operatorname{id}_{A} I<\infty$.

The next result is a direct consequence of Theorem (2.1).

Corollary (2.3). Let $A$ be a Noetherian local ring with the maximal ideal $\mathfrak{m}$. Then $A$ is a regular local ring if $A$ contains an integrally closed $\mathfrak{m}$-primary ideal possessing finite injective dimension. In particular $A$ is a regular local ring if and only if $\operatorname{id}_{A} \mathfrak{m}<\infty$.

We now prove the following. It was inspired by a discussion with K. Yoshida of Nagoya University.

Corollary (2.4). Let $A$ be a Cohen-Macaulay local ring possessing the canonical module $\mathrm{K}_{A}$. Assume that $A$ contains an ideal $I(\neq A)$ such that $I \cong \mathrm{K}_{A}$. Then $A$ is a regular local ring if $A / I$ is regular.

Proof. Let $d=\operatorname{dim} A$. Then $d>0$ and $\operatorname{dim} A / I=d-1$ ([HK, Korollar 6.13]). Therefore, if $d=1$, then $I=\mathfrak{m}$, so that by Corollary (2.3) $A$ must be a DVR (recall that $\left.\operatorname{id}_{A} I=\operatorname{id}_{A} \mathrm{~K}_{A}<\infty\right)$. Suppose that $d>1$ and our assertion holds true for $d-1$. We choose an element $f \in \mathfrak{m} \backslash\left(\mathfrak{m}^{2}+I\right)$ so that $f \notin \bigcup_{\mathfrak{p} \in \operatorname{Ass} A} \mathfrak{p}$. Then, since $f$ is regular both on $A$ and $A / I$, we get the isomorphisms

$$
\mathrm{K}_{A / f A} \cong \mathrm{K}_{A} / f \mathrm{~K}_{A} \text { and } I / f I \cong(I+f A) / f A
$$

(see [HK, Korollar 6.3] for the first isomorphism). Thus the hypothesis on $d$ guarantees the local ring $A / f A$ is regular, so that $A$ is a regular local ring.

\section{Proof of Theorem (1.1) And some consequences}

Let $A$ be a regular local ring with the maximal ideal $\mathfrak{m}$ and $d=\operatorname{dim} A$. Let $\mathcal{F}=\left\{F_{n}\right\}_{n \in \mathbb{Z}}$ be a good filtration of ideals in $A$. We put $\mathcal{R}=\mathcal{R}(\mathcal{F}), \mathcal{R}^{\prime}=\mathcal{R}^{\prime}(\mathcal{F})$, and $\mathcal{G}=\mathcal{G}(\mathcal{F})$. Let $\mathfrak{M}=\mathfrak{m} \mathcal{R}+\mathcal{R}_{+}$denote the graded maximal ideal in $\mathcal{R}$.

Proof of Theorem (1.1). (1) $\Leftrightarrow$ (3) This follows from the fact that $\mathcal{G}=\mathcal{R}^{\prime} / t^{-1} \mathcal{R}^{\prime}$ and the isomorphism $\mathrm{K}_{\mathcal{G}} \cong\left[\mathrm{K}_{\mathcal{R}^{\prime}} / t^{-1} \mathrm{~K}_{\mathcal{R}^{\prime}}\right]$ (-1) (cf. [GW], Remark (3.1.6)]; recall that $t^{-1}$ is a non-zerodivisor of $\mathcal{R}^{\prime}$ ).

$(1) \Rightarrow(2)$ The ring $\mathcal{R}$ is Cohen-Macaulay ([GNi Part II, Corollary (1.2)]), while $\mathcal{R}^{\prime}$ is a Gorenstein ring with $\mathrm{K}_{\mathcal{R}^{\prime}} \cong \mathcal{R}^{\prime}$ as graded $\mathcal{R}^{\prime}$-modules. Hence the canonical $\mathcal{F}$-filtration $\omega$ of $A$ in the sense of GI coincides with $\mathcal{F}$ itself, so that we have $\mathrm{K}_{\mathcal{R}} \cong \mathcal{R}_{+}$as graded $\mathcal{R}$-modules (cf. [GI, Proposition 2.5]). 
(2) $\Rightarrow$ (3) Since $\mathrm{K}_{\mathcal{R}}=\mathcal{R}_{+}$, identifying $\mathcal{R}_{+}(1)=t^{-1} \mathcal{R}^{\prime} \cap \mathcal{R}$ we get the exact sequence

$$
0 \rightarrow\left[\mathrm{K}_{\mathcal{R}}\right](1) \rightarrow \mathcal{R} \rightarrow \mathcal{G} \rightarrow 0
$$

of graded $\mathcal{R}$-modules. Hence $\mathcal{G}$ is a Gorenstein ring by [HK Korollar 6.13]. By sequence $(*)$ we also have the embedding

$$
0 \rightarrow \mathrm{H}_{\mathfrak{M}}^{d}(\mathcal{G}) \rightarrow \mathrm{H}_{\mathfrak{M}}^{d+1}\left(\mathrm{~K}_{\mathcal{R}}\right)(1)
$$

of local cohomology modules. Hence the socle $\left[(0):_{\mathrm{H}_{\mathfrak{M}}^{d}}(\mathcal{G}) \mathfrak{M}\right]$ of $\mathrm{H}_{\mathfrak{M}}^{d}(\mathcal{G})$ is concentrated in degree -1 , because $\mathrm{H}_{\mathfrak{M}}^{d+1}\left(\mathrm{~K}_{\mathcal{R}}\right)=\mathrm{E}_{\mathcal{R}}(\mathcal{R} / \mathfrak{M})$ (the injective envelope of $\mathcal{R} / \mathfrak{M})$ and the socle of $\mathrm{E}_{\mathcal{R}}(\mathcal{R} / \mathfrak{M})$ is concentrated in degree 0 . Hence $\mathrm{a}(\mathcal{G})=-1$.

We now assume that $A$ is a regular local ring.

$(2) \Rightarrow(4)$ The local ring $\mathcal{R}_{\mathfrak{M}}$ must be regular by Corollary (2.4), since $A=$ $\mathcal{R} / \mathcal{R}_{+}=\mathcal{R} / \mathrm{K}_{\mathcal{R}}$ is a regular local ring. Hence the ring $\mathcal{R}$ is regular.

$(4) \Rightarrow(6)$ We have $\ell_{A}\left(\left(\mathfrak{m} \mathcal{R}+\mathfrak{M}^{2}\right) / \mathfrak{M}^{2}\right)=d$ since $\mathfrak{M}^{2} \cap A=\mathfrak{m}^{2}$ (here $\ell_{A}(*)$ stands for the length). Therefore, since $\ell_{A}\left(\mathfrak{M} / \mathfrak{M}^{2}\right)=\operatorname{dim} \mathcal{R}_{\mathfrak{M}}=d+1$ and $F_{1} t \nsubseteq$ $\mathfrak{m} \mathcal{R}+R_{+}^{2}=\mathfrak{m} \mathcal{R}+\mathfrak{M}^{2}$, we may choose an element $x \in F_{1}$ so that $\mathfrak{M}=\mathfrak{m} \mathcal{R}+x t \cdot \mathcal{R}$. Let $n>0$ be an integer. Then, since $F_{n} t^{n} \subseteq \mathfrak{M}=\mathfrak{m} \mathcal{R}+x t \cdot \mathcal{R}$, we have $F_{n}=$ $\mathfrak{m} F_{n}+x F_{n-1}$; hence $F_{n}=x F_{n-1}$ by Nakayama's lemma. Consequently $F_{n}=\left(x^{n}\right)$ for all $n>0$.

$(6) \Rightarrow(5)$ and (3) Let $F_{1}=(x)$ with $0 \neq x \in F_{1}$ and put $T=x t$. Then $\mathcal{R}=A[T]$ is the polynomial ring. Therefore $\mathcal{G}=\mathcal{R} / x \mathcal{R}$ is also the polynomial ring in one variable over $A /(x)$, so that $\mathcal{G}$ is a Gorenstein ring with a $(\mathcal{G})=-1$ (GW, Remark (3.1.6)]).

$(5) \Rightarrow(4)$ This implication is clear.

Remark (3.1). Unless $A$ is regular, the implication $(3) \Rightarrow(6)$ in Theorem (1.1) is no longer true, even though $A$ is a rational singularity. The simplest example is as follows. Let $R=k[[X, Y, Z]]$ be the formal power series ring in three variables over a field $k$. We put $A=R /\left(Z^{2}-X Y\right)$ and take $I=\mathfrak{m}$, the maximal ideal in $A$. Then $A$ is a 2-dimensional rational singularity and $\mathcal{G}(\mathfrak{m})=k[X, Y, Z] /\left(Z^{2}-X Y\right)$, where $k[X, Y, Z]$ denotes the polynomial ring over $k$. Hence $\mathcal{G}(\mathfrak{m})$ is a Gorenstein ring but a $(\mathcal{G}(\mathfrak{m}))=-1$. See [GIW, Section 7] for further examples.

Remark (3.2). Even though the ideal $F_{1}$ is principal and the ring $\mathcal{G}(\mathcal{F})$ is Gorenstein, the filtration $\mathcal{F}$ is not necessarily ideal-adic, so that the inequality a $(\mathcal{G}(\mathcal{F})) \leq$ -2 may hold true. For instance, let $A$ be a regular local $\operatorname{ring}$ of $\operatorname{dim} A \geq 1$ and choose $\pi \in \mathfrak{m} \backslash \mathfrak{m}^{2}$. Let $\ell \geq 1$ be an integer. For each $0<n \in \mathbb{Z}$ we put $F_{n}=\pi^{q+1} A$, where $q=\max \left\{q \in \mathbb{Z} \mid q<\frac{n}{\ell}\right\}$. Then, letting $F_{n}=A$ for $n \leq 0$, the family $\mathcal{F}=\left\{F_{n}\right\}_{n \in \mathbb{Z}}$ forms a filtration of ideals in $A$. We have $\mathcal{R}(\mathcal{F})=A\left[\left\{\pi t^{n}\right\}_{1 \leq n \leq \ell}\right]$ and $\mathcal{R}^{\prime}(\mathcal{F})=A\left[\pi t^{\ell}, t^{-1}\right]$. Therefore $\mathcal{G}(\mathcal{F})=B[T]$ is the polynomial ring in one variable $T\left(=\pi t^{\ell} \bmod t^{-1} \mathcal{R}^{\prime}(\mathcal{F})\right)$ of degree $\ell$ over the regular local ring $B=A / \pi A$; hence a $(\mathcal{G}(\mathcal{F}))=-\ell$. If $\ell \geq 2$, we have $F_{2} \neq F_{1}^{2}$ since $F_{1}=F_{2}=\pi A$. The ring $\mathcal{R}(\mathcal{F})$ is not regular but Gorenstein.

\section{Ideals of SMALL Height}

In this section let us gather some consequences of Theorem (1.1) in the case where $F_{n}=I^{n}(n \in \mathbb{Z})$ for some ideal $I$ of small height. We first note the following. 
Corollary (4.1). Let $A$ be a regular local ring and let $I$ be an ideal in $A$ of $\mathrm{ht}_{A} I=$ 1. Then $\mathcal{G}(I)$ is a Gorenstein ring if and only if $I$ is a principal ideal.

Proof. It suffices to check the only if part. Choose $\mathfrak{p} \in \mathrm{V}(I)$ so that $\mathrm{ht}_{A} \mathfrak{p}=1$. Then a $(\mathcal{G}(I))=\mathrm{a}\left(A_{\mathfrak{p}} \otimes_{A} \mathcal{G}(I)\right)=\mathrm{a}\left(\mathcal{G}\left(I A_{\mathfrak{p}}\right)\right)$, since $\mathcal{G}(I)$ is a Gorenstein ring. Hence $\mathrm{a}(\mathcal{G}(I))=-1$, because $I A_{\mathfrak{p}}$ is principal. Thus $I$ must be principal by Theorem (1.1).

Corollary (4.2) ([HHR, Theorem 2.5]). Let $A$ be a regular local ring and let $I$ be an ideal in $A$ of $\mathrm{ht}_{A} I=s \geq 2$. Assume that $I$ contains a reduction $\mathfrak{q}$ generated by $s$ elements. Then $I^{s-1}=\mathfrak{q} I^{s-2}$ if $\mathcal{G}(I)$ is a Gorenstein ring. Hence $I=\mathfrak{q}$ if $\mathcal{G}(I)$ is Gorenstein and $s=2$.

Proof. Let $\mathrm{r}_{\mathfrak{q}}(I)=\min \left\{0 \leq n \in \mathbb{Z} \mid I^{n+1}=\mathfrak{q} I^{n}\right\}$. Then since $\mathcal{G}(I)$ is CohenMacaulay and $I$ is equi-multiple, we have a $(\mathcal{G}(I))=\mathrm{r}_{\mathfrak{q}}(I)-s$ (cf., e.g., GI Preface of Section 5]). Hence $\mathrm{r}_{\mathfrak{q}}(I) \leq s-2$, because a $(\mathcal{G}(I)) \leq-2$ by Corollary (1.2).

Corollary (4.3). Let $A$ be a 2-dimensional regular local ring. Let I be an ideal in $A$ and assume that $\mathcal{G}(I)$ is a Gorenstein ring. Then $I$ is a complete intersection.

Proof. The assertion directly follows from Corollaries (4.1) and (4.2) (enlarge the residue class field of $A$ if necessary).

We now focus our attention on the ideals $I$ of $\mathrm{ht}_{A} I=2$.

Corollary (4.4). Let $I$ be an ideal of $\mathrm{ht}_{A} I=2$ in a regular local ring $A$. Then the following two conditions are equivalent:

(1) $\mathcal{R}(I)$ is a Gorenstein ring.

(2) $\mathcal{G}(I)$ is a Gorenstein ring.

When this is the case, $I$ is generically a complete intersection and $\mathrm{a}(\mathcal{G}(I))=-2$.

Proof. (1) $\Rightarrow(2)$ See [I, Theorem (3.1)].

$(2) \Rightarrow(1)$ Choose $\mathfrak{p} \in \mathrm{V}(I)$ with $\mathrm{ht}_{A} \mathfrak{p}=2$. Then by Corollary (4.3) I $A_{\mathfrak{p}}$ is generated by an $A_{\mathfrak{p}}$-regular sequence of length 2 , since $\mathcal{G}\left(I A_{\mathfrak{p}}\right)$ is a Gorenstein ring. Thus a $(\mathcal{G}(I))=\mathrm{a}\left(\mathcal{G}\left(I A_{\mathfrak{p}}\right)\right)=-2$, so that by [I, Theorem $\left.(3.1)\right] \mathcal{R}(I)$ is a Gorenstein ring.

Let $\lambda(I)=\operatorname{dim} A / \mathfrak{m} \otimes_{A} \mathcal{R}(I)$ denote the analytic spread of $I$.

Corollary (4.5). Let $A$ be a regular local ring with the infinite residue class field. Let $I$ be an ideal in $A$ with $\mathrm{ht}_{A} I=2$ and $\lambda(I)=3$. Let

$$
U=\bigcap_{\mathfrak{p} \in \mathrm{V}(I) \text { such that } \mathrm{ht}_{A} \mathfrak{p}=2}\left(I A_{\mathfrak{p}} \cap A\right)
$$

be the unmixed component and $J$ a minimal reduction of $I$. Then the following three conditions are equivalent:

(1) $\mathcal{R}(I)$ is a Gorenstein ring.

(2) $\mathcal{G}(I)$ is a Gorenstein ring.

(3) $I$ is generically a complete intersection such that $\operatorname{depth} A / I \geq d-3, I^{2}=J I$, and $(J U: I) \cap U=I$.

When this is the case, one has the equality $\mathrm{a}(\mathcal{G}(I))=-2$.

Proof. See Corollary (4.4) and [GI, Theorem 6.4]. 
It might be interesting to classify the ideals $I$ of $\mathrm{ht}_{A} I=2$ in a regular local $\operatorname{ring} A$ for which $\mathcal{G}(I)$ are Gorenstein rings and a $(\mathcal{G}(I))=-2$. The 2-dimensional case is done with Corollary (4.3). The situation is more complicated in the case where $\operatorname{dim} A=3$. By Corollary (4.4) our ideal $I$ has to be generically a complete intersection with $\lambda(I) \leq \operatorname{dim} A=3$. Therefore, if $A / I$ is Cohen-Macaulay, $I$ is either a complete intersection or almost a complete intersection generated by a $d$ sequence of length 3 (GNa, Theorem (1.3)]). We precisely have $\mu_{A}(I)=\ell_{A}((I$ : $\mathfrak{m}) / I)+3\left(\right.$ GNa , Theorem (3.8)]) if $I$ is not a complete intersection, where $\mu_{A}(I)$ denotes the number of generators of $I$. However, every regular local ring $A$ of $\operatorname{dim} A=3$ contains numerous non-Cohen-Macaulay ideals $I$ of ht ${ }_{A} I=2$ such that the rings $\mathcal{G}(I)$ are Gorenstein, and except for Corollary (4.5) the authors do not know how to manage them. Concluding this paper, we note Example (4.6) for such ideals.

Let $A$ be a regular local $\operatorname{ring}$ of $d=\operatorname{dim} A \geq 3$. Let $X, Y, Z$ be a subsystem of parameters in $A$ and choose integers $\alpha, \beta$ so that $2 \alpha \geq \beta>\alpha>0$. We put $I=$ $\left(X^{2}, Y^{\alpha}\right) \cap\left(X, Y^{\beta}, Z\right)\left(=\left(X^{2}, Y^{\beta}, X Y^{\alpha}, Y^{\alpha} Z\right)\right)$. Then $\mathrm{ht}_{A} I=2$ and $\mu_{A}(I)=4$. We furthermore have the following.

Example (4.6). The following assertions hold true:

(1) $\operatorname{dim} A / I=d-2$, depth $A / I=d-3$, and $\lambda(I)=3$.

(2) $\mathcal{G}(I)$ is a Gorenstein ring and a $(\mathcal{G}(I))=-2$.

Proof. Enlarging the residue class field of $A$, we may assume that the field $A / \mathfrak{m}$ is infinite. Let $U=\left(X^{2}, Y^{\alpha}\right)$ and $J=\left(X^{2}, Y^{\beta}, X Y^{\alpha}+Y^{\alpha} Z\right)$. Then, considering the exact sequence

$$
0 \rightarrow A / I \rightarrow A / U \oplus A /\left(X, Y^{\beta}, Z\right) \rightarrow A /\left(X, Y^{\alpha}, Z\right) \rightarrow 0
$$

of $A$-modules, we get that $\operatorname{depth} A / I \geq d-3$ (use the depth lemma). Since $\operatorname{dim} A / I=d-2$ and the ideal $I$ is mixed, the local ring $A / I$ cannot be CohenMacaulay, whence depth $A / I=d-3$. We have $J \subseteq I$ and $I^{2}=J I$. Hence $\lambda(I) \leq 3$. Because $A / I$ is not a Cohen-Macaulay ring but the ideal $I$ is generically a complete intersection, we see $\lambda(I)>2$, so that $\lambda(I)=3$ whence $J$ is a minimal reduction of $I$. It is routine and standard to check that $(J U: I) \cap U=I$. Thus assertion (2) directly follows from Corollary (4.5), because $U$ is the unmixed component of the ideal $I$.

A similar argument also works to show that $\mathcal{G}(I)$ is a Gorenstein ring for the non-Cohen-Macaulay ideal $I=\left(X^{2}, Y Z\right) \cap\left(X, Y^{2}, Z^{\alpha}\right)\left(=\left(X^{2}, X Y Z, Y^{2} Z, Y Z^{\alpha}\right)\right)$ $(\alpha \geq 2)$ in $A$.

\section{REFERENCES}

[B] H. Bass, On the ubiquity of Gorenstein rings, Math. Z. 82 (1963), 8-28. MR 27:3669

[FT] H.-B. Foxby and A. Thorup, Minimal injective resolutions under flat base changes, Proc. Amer. Math. Soc. 67 (1977), 27-31. MR 56:11984

[G] S. Goto, Integral closedness of complete-intersection ideals, J. Alg. 108 (1987), 151-160. MR 88d:13015

[GH] S. Goto and F. Hayasaka, Finite homological dimension and primes associated to integrally closed ideals, Proc. Amer. Math. Soc. (to appear).

[GI] S. Goto and S.-i. Iai, Embedding of certain graded rings into their canonical modules, J. Alg. 228 (2000), 377-396. MR 2001f: 13006

[GIW] S. Goto, S.-i. Iai, and K.-i. Watanabe, Good ideals in Gorenstein local rings, Trans. Amer. Math. Soc. (2000), 2309-2346. CMP 2001:08 
[GK] S. Goto and M.-k. Kim, Equimultiple good ideals, J. Math. Kyoto Univ. 42-1 (2002), 21-32.

[GNa] S. Goto and Y. Nakamura, On the Gorensteinness of graded rings associated to ideals of analytic deviation one, Contemporary Mathematics 159 (1994), 51-72.

[GNi] S. Goto and K. Nishida, The Cohen-Macaulay and Gorenstein properties of Rees algebras associated to filtrations, Mem. Amer. Math. Soc., 110, 1994. MR 95b:13001

[GNS1] S. Goto, K. Nishida, and Y. Shimoda, The Gorensteinness of the symbolic Rees algebras for space curves, J. Math. Soc. Japan 43 (1991), 465-481. MR 92h:13019

[GNS2] S. Goto, K. Nishida, and Y. Shimoda, The Gorensteinness of the symbolic blow-ups for certain space monomial curves, Trans. Amer. Math. Soc. 340 (1993), 323-335. MR 94a:13002

[GS] S. Goto and Y. Shimoda, On the Rees algebras of Cohen-Macaulay local rings, Commutative Algebra, Analytic Methods (R. N. Draper, ed.), Lecture Notes in Pure and Applied Mathematics, vol. 68, Marcel Dekker, Inc., New York and Basel, 1982, pp. 201-231. MR 84a:13021

[GW] S. Goto and K. Watanabe, On graded rings, I, J. Math. Soc. Japan 30 (1978), 179-213. MR 81m:13021

[HHR] M. Herrmann, C. Huneke, and J. Ribbe, On reduction exponents of ideals with Gorenstein form rings, Proc. Edinburgh Math. Soc. 38 (1995), 449-463. MR 96i:13007

[HIO] M. Herrmann, S. Ikeda, and U. Orbanz, Equimultiplicity and Blowing Up, SpringerVerlag, 1988. MR 89g:13012

[HK] J. Herzog and E. Kunz (eds.), Der kanonische Modul eines Cohen-Macaulay-Rings, Lecture Notes in Math., vol. 238, Springer-Verlag, Berlin · Heidelberg · New York · Tokyo, 1971. MR 54:304

[I] S. Ikeda, On the Gorensteinness of Rees algebras over local rings, Nagoya Math. J. 102 (1986), 135-154. MR 87j:13031

[L] J. Lipman, Cohen-Macaulayness in graded algebras, Math. Res. Lett. 1 (1994), 149-157. MR 95d:13006

[TI] N. V. Trung and S. Ikeda, When is the Rees algebra Cohen-Macaulay?, Comm. Alg. 17 (1989), 2893-2922. MR 91a:13009

Department of Mathematics, School of Science and Technology, Meiji University, KAWASAKI, 214-8571 JAPAN

E-mail address: goto@math.meiji.ac.jp

Department of Mathematics, School of Science and Technology, Meiji University, KAWASAKI, 214-8571 JAPAN

E-mail address: ee68048@math.meiji.ac.jp

Department of Mathematics, Hokkaido University of Education, Sapporo, 002-8502 JAPAN

E-mail address: iai@sap.hokkyodai.ac.jp 